\title{
A Phragmén-Lindelöf Theorem via Proximate Orders, and the Propagation of Asymptotics
}

\author{
Javier Jiménez-Garrido ${ }^{1}$ - Javier Sanz ${ }^{1}$ - Gerhard Schindl ${ }^{2}$
}

Received: 13 September 2018 / Published online: 10 May 2019

(c) The Author(s) 2019

\begin{abstract}
We prove that, for asymptotically bounded holomorphic functions in a sector in $\mathbb{C}$, an asymptotic expansion in a single direction towards the vertex with constraints in terms of a logarithmically convex sequence admitting a nonzero proximate order entails asymptotic expansion in the whole sector with control in terms of the same sequence. This generalizes a result by Fruchard and Zhang for Gevrey asymptotic expansions, and the proof strongly rests on a suitably refined version of the classical Phragmén-Lindelöf theorem, here obtained for functions whose growth in a sector is specified by a nonzero proximate order in the sense of Lindelöf and Valiron.
\end{abstract}

Keywords Asymptotic expansion - Ultraholomorphic functions · Nonzero proximate orders · Phragmén-Lindelöf theorem

Mathematics Subject Classification $30 \mathrm{E} 15 \cdot 30 \mathrm{C} 80 \cdot 26 \mathrm{~A} 12 \cdot 30 \mathrm{H} 50$

\section{Introduction}

In 1999, Fruchard and Zhang [3] proved that, for a holomorphic function in a sector $S$ which is bounded in every proper subsector of $S$, the existence of an asymptotic expansion following just one direction implies global (non-uniform) asymptotic expansion

$\bowtie \quad$ Gerhard Schindl

gerhard.schindl@univie.ac.at

Javier Jiménez-Garrido

jjjimenez@am.uva.es

Javier Sanz

jsanzg@am.uva.es

1 Departamento de Álgebra, Análisis Matemático, Geometría y Topología, Instituto de Investigación en Matemáticas de la Universidad de Valladolid, IMUVA, Facultad de Ciencias, Universidad de Valladolid, 47011 Valladolid, Spain

2 Fakultät für Mathematik, Universität Wien, Oskar-Morgenstern Platz 1, 1090 Vienna, Austria 
in the whole of $S$. Moreover, a Gevrey version of this result is provided with a control on the type as below:

Theorem 1.1 ([3], Theorem 11) Let $f$ be a function analytic and bounded in an open sector $S=S(d, \gamma, r)$ of bisecting direction $d \in \mathbb{R}$, opening $\pi \gamma$ and radius $r$, with $\gamma, r>0$. Suppose $f$ has asymptotic expansion $\hat{f}=\sum_{n=0}^{\infty} a_{n} z^{n}$ of Gevrey order $1 / k(k>0)$ and type (at least) $R\left(\theta_{0}\right)>0$ in some direction $\theta_{0}$ with $\left|\theta_{0}-d\right|<\pi \gamma / 2$, i.e., for every $\delta>0$, there exists $C=C(\delta)>0$ such that for every $z \in S$ with $\arg (z)=\theta_{0}$ and every nonnegative integer $p$, we have that

$$
\left|f(z)-\sum_{n=0}^{p-1} a_{n} z^{n}\right| \leq C\left(\frac{1}{R\left(\theta_{0}\right)}+\delta\right)^{p} \Gamma\left(1+\frac{p}{k}\right)|z|^{p} .
$$

Then, in every direction $\theta$ of $S, f$ admits $\hat{f}$ as its asymptotic expansion of Gevrey order $1 / k$ and type $R(\theta)$ given as follows:

$$
R(\theta)= \begin{cases}R\left(\theta_{0}\right)\left(\frac{\sin k(\theta-\alpha)}{\sin k\left(\alpha^{\prime}-\alpha\right)}\right)^{1 / k} & \text { if } \theta \in\left(\alpha, \alpha^{\prime}\right], \\ R\left(\theta_{0}\right) & \text { if } \theta \in\left[\alpha^{\prime}, \beta^{\prime}\right], \\ R\left(\theta_{0}\right)\left(\frac{\sin k(\theta-\beta)}{\sin k\left(\beta^{\prime}-\beta\right)}\right)^{1 / k} & \text { if } \theta \in\left[\beta^{\prime}, \beta\right) .\end{cases}
$$

Here, $\alpha=d-\pi \gamma / 2$ and $\beta=d+\pi \gamma / 2$ are the directions of the radial boundary of $S, \alpha^{\prime}=\min \left(\theta_{0}, \alpha+\frac{\pi}{2 k}\right) \in\left(\alpha, \theta_{0}\right]$, and $\beta^{\prime}=\max \left(\theta_{0}, \beta-\frac{\pi}{2 k}\right) \in\left[\theta_{0}, \beta\right)$.

We warn the reader that there is no agreement about the terminology in this respect: while most authors adhere, as we will do, to the convention that the asymptotics in (1.1) is Gevrey of order $1 / k$, others (for example, Fruchard and Zhang or Balser [1]) say this is of order $k$. Moreover, the notion of type is not standard, as compared with the definition by Canalis-Durand [2] for whom the type in case one has $(1.1)$ is $(1 / R+\delta)^{k}$. It should also be mentioned that the factor $\Gamma(1+p / k)$ could be changed into $(p !)^{1 / k}$ without changing the asymptotics, but this would affect the base of the geometric factor providing the type (by Stirling's formula, [2, pp. 3-4]) in any case. As will be explained later, our interest in the type will be limited, and so we will choose a simple approach in this respect-see Definitions 2.2 and 2.11.

The proof of Theorem 1.1 is based on the classical Phragmén-Lindelöf theorem and on the so-called Borel-Ritt-Gevrey theorem. This last statement provides the surjectivity, as long as the opening of the sector is at most $\pi / k$, of the Borel map, sending a function with Gevrey asymptotic expansion of order $1 / k$ in that sector to its series of asymptotic expansion (coefficients of which will necessarily satisfy Gevreylike estimates). Also, the injectivity of the Borel map in sectors of opening greater than $\pi / k$ (known as Watson's lemma) plays an important role when guaranteeing the uniqueness of a function with a prescribed Gevrey asymptotic expansion of order $1 / k$ in a direction.

The main aim of this paper is to extend these results for other types of asymptotic expansions available in the literature. This possibility was already mentioned in 
[12], where Lastra, Mozo-Fernández, and the second author of this paper generalized the results of Fruchard and Zhang to the several variables setting. They considered holomorphic functions in a polysector (cartesian product of sectors) admitting strong asymptotic expansion in the sense of Majima [14,15], also in the Gevrey case as introduced by Haraoka [5].

We will deal with general ultraholomorphic classes of functions, defined by constraining the growth of their derivatives in a sector in terms of a sequence $\mathbb{M}=$ $\left(M_{p}\right)_{p \in \mathbb{N}_{0}}$ of positive numbers $\left(\mathbb{N}_{0}=\{0,1,2, \ldots\}=\{0\} \cup \mathbb{N}\right)$, see Definition 2.3. This sequence will play the role of $(\Gamma(1+p / k))_{p \in \mathbb{N}_{0}}$ in (1.1). It will be subject to precise conditions in order to guarantee not only the natural algebraic and analytic properties of the corresponding class, but also the possibility of extending to this more general framework the results on the injectivity or surjectivity of the Borel map and a Phragmén-Lindelöf-like statement. The relation of these classes to those of functions with an asymptotic expansion is extremely close, see Proposition 2.4.

For log-convex sequences $\mathbb{M}$, the considered ultraholomorphic classes are algebras. The injectivity of the Borel map had been characterized in the 1950 s by Mandelbrojt [16] for uniform asymptotics (see Theorem 2.19 in this paper) and by Rodríguez-Salinas [17] for uniformly bounded derivatives (see Theorem 2.15 here). However, regarding surjectivity only some partial results were available by Schmets and Valdivia [19] and Thilliez [20] at the very beginning of this century. They rested on results from the ultradifferentiable setting (dealing with classes of smooth functions in open sets of $\mathbb{R}^{n}$ with suitably controlled derivatives), and disregarded questions about the optimality of the opening of the sector or the variation of the type along with the direction in the sector. Moreover, the techniques used, of a functional-analytic nature, do not provide any insight into a possible extension of the Phragmén-Lindelöf theorem. However, the second author [18] has recently made intervene the classical concept of proximate order in these concerns, making possible to obtain more precise statements concerning the injectivity and surjectivity of the Borel map. Subsequently, the authors $[8,9]$ have studied the relationship between log-convex sequences, nonzero proximate orders and the property of regular variation. As a result, a deeper understanding has been gained of the property of admissibility of a nonzero proximate order by a log-convex sequence. This is the key for obtaining in this paper an analog of Phragmén-Lindelöf theorem for functions whose growth in a sector is specified in terms of such a sequence $\mathbb{M}$. It is worth mentioning that sequences admitting a nonzero proximate order are strongly regular (in the sense of Thilliez), and that all the instances of strongly regular sequences appearing in applications do admit such a proximate order.

As in the Gevrey case, the study of the type as the direction moves in the sector is possible, although some information is lost in general (see Remark 3.3). This is due to the fact that the classical exponential kernel, appearing in a suitable truncated Laplace transform providing the solution of the Borel-Ritt-Gevrey theorem in the Gevrey case, is now replaced by the exponential of a function whose behavior at infinity is only given by some asymptotic relations. However, in case the sequence $\mathbb{M}$ not only admits a nonzero proximate order, but provides one, the type may be better described. 
The paper is organized as follows. After fixing some notations, Sect. 2 is devoted to some preliminaries on general asymptotic expansions, ultraholomorphic classes and quasianalyticity results, specially when nonzero proximate orders are available. All this material will be needed in Sect. 3, where several lemmas of a Phragmén-Lindelöf flavor are obtained. A paradigm is Lemma 3.2, where exponential decrease is extended from just one direction to a whole small (in the sense of its opening) sector adjacent to it. Section 4 contains several versions of Watson's lemma on the uniqueness of a function admitting a given asymptotic expansion in a direction, and in the final Sect. 5, we characterize the functions with an asymptotic expansion in a sectorial region as those asymptotically bounded and admitting such expansion in just one direction in the region.

The results presented in this paper are part of the Ph.D. Dissertation of the first author [7], defended at the University of Valladolid (Spain) under the advice of the second author.

\section{Preliminaries}

We set $\mathbb{N}:=\{1,2, \ldots\}, \mathbb{N}_{0}:=\mathbb{N} \cup\{0\}$. $\mathcal{R}$ stands for the Riemann surface of the logarithm. We consider bounded sectors

$$
S(d, \gamma, r):=\left\{z \in \mathcal{R}:|\arg z-d|<\frac{\gamma \pi}{2},|z|<r\right\}
$$

respectively, unbounded sectors

$$
S(d, \gamma):=\left\{z \in \mathcal{R}:|\arg z-d|<\frac{\gamma \pi}{2}\right\}
$$

with bisecting direction $d \in \mathbb{R}$, opening $\gamma \pi(\gamma>0)$, and (in the first case) radius $r \in(0, \infty)$. For unbounded sectors of opening $\gamma \pi$ bisected by direction 0 , we write $S_{\gamma}:=S(0, \gamma)$. In some cases, it will also be convenient to consider sectors whose elements have their argument in a half-open, or in a closed, bounded interval of the real line.

A sectorial region $G(d, \gamma)$ with bisecting direction $d \in \mathbb{R}$ and opening $\gamma \pi$ will be an open connected set in $\mathcal{R}$ such that $G(d, \gamma) \subset S(d, \gamma)$, and for every $\beta \in(0, \gamma)$ there exists $\rho=\rho(\beta)>0$ with $S(d, \beta, \rho) \subset G(d, \gamma)$. In particular, sectors are sectorial regions. If $d=0$, we just write $G_{\gamma}$.

A bounded (respectively, unbounded) sector $T$ is said to be a proper subsector of a sectorial region (resp. of an unbounded sector) $G$, and we write $T \ll G$ (resp. $T \prec G$ ), if $\bar{T} \subset G$ (where the closure of $T$ is taken in $\mathcal{R}$, and so the vertex of the sector is not under consideration).

For an open set $U \subset \mathcal{R}$, the set of all holomorphic functions in $U$ will be denoted by $\mathcal{H}(U) . \mathbb{C}[[z]]$ stands for the set of formal power series in $z$ with complex coefficients. 


\subsection{Log-convex Sequences and Ultraholomorphic Classes}

In what follows, $\mathbb{M}=\left(M_{p}\right)_{p \in \mathbb{N}_{0}}$ always stands for a sequence of positive real numbers, and we always assume that $M_{0}=1$.

Definition 2.1 We say a holomorphic function $f$ in a sectorial region $G$ admits the formal power series $\hat{f}=\sum_{n=0}^{\infty} a_{n} z^{n} \in \mathbb{C}[[z]]$ as its $\mathbb{M}$-asymptotic expansion in $G$ (when the variable tends to 0 ) if for every $T \ll G$ there exist $C_{T}, A_{T}>0$ such that for every $p \in \mathbb{N}_{0}$ one has

$$
\left|f(z)-\sum_{n=0}^{p-1} a_{n} z^{n}\right| \leq C_{T} A_{T}^{p} M_{p}|z|^{p}, \quad z \in T .
$$

We will write $f \sim_{\mathbb{M}} \hat{f}$ in $G$, and $\tilde{\mathcal{A}}_{\mathbb{M}}(G)$ will stand for the space of functions admitting M-asymptotic expansion in $G$.

Definition 2.2 Given a sector $S$, we say $f \in \mathcal{H}(S)$ admits $\hat{f}=\sum_{n=0}^{\infty} a_{n} z^{n} \in \mathbb{C}[[z]]$ as its uniform $\mathbb{M}$-asymptotic expansion in $S$ (of type $1 / A$ for some $A>0$ ) if there exists $C>0$ such that for every $p \in \mathbb{N}_{0}$ one has

$$
\left|f(z)-\sum_{n=0}^{p-1} a_{n} z^{n}\right| \leq C A^{p} M_{p}|z|^{p}, \quad z \in S
$$

$\tilde{\mathcal{A}}_{\mathbb{M}}^{\mathrm{u}}(S)$ stands for the space of functions admitting uniform $\mathbb{M}$-asymptotic expansion in $S$ (of some type).

Definition 2.3 Given $\mathbb{M}=\left(M_{p}\right)_{p \in \mathbb{N}_{0}}$, a constant $A>0$ and a sector $S$, we define

$$
\mathcal{A}_{\mathbb{M}, \mathrm{A}}(S)=\left\{f \in \mathcal{H}(S):\|f\|_{\mathbb{M}, \mathrm{A}}:=\sup _{z \in S, n \in \mathbb{N}_{0}} \frac{\left|f^{(p)}(z)\right|}{A^{p} p ! M_{p}}<\infty\right\} .
$$

$\left(\mathcal{A}_{\mathbb{M}, \mathrm{A}}(S),\|\|_{\mathbb{M}, \mathrm{A}}\right)$ is a Banach space, and $\mathcal{A}_{\mathbb{M}}(S):=\cup_{A>0} \mathcal{A}_{\mathbb{M}, \mathrm{A}}(S)$ is called a Roumieu-Carleman ultraholomorphic class in the sector $S$.

Since the derivatives of $f \in \mathcal{A}_{\mathbb{M}, \mathrm{A}}(S)$ are Lipschitzian, for every $n \in \mathbb{N}_{0}$ one may define

$$
f^{(n)}(0):=\lim _{z \in S, z \rightarrow 0} f^{(n)}(z) \in \mathbb{C} .
$$

We recall now the relationship between these classes and the concept of asymptotic expansion. As a consequence of Taylor's formula, we have the following result (see $[1,4])$. 
Proposition 2.4 Let $S$ be a sector, if $f \in \mathcal{A}_{\mathbb{M}, \mathrm{A}}(S)$ then $f$ admits $\hat{f}=$ $\sum_{p \in \mathbb{N}_{0}} \frac{1}{p !} f^{(p)}(0) z^{p}$ as its uniform $\mathbb{M}$-asymptotic expansion in $S$ of type $1 / A$. Consequently, we have that

$$
\mathcal{A}_{\mathbb{M}}(S) \subseteq \tilde{\mathcal{A}}_{\mathbb{M}}^{\mathrm{u}}(S) \subseteq \tilde{\mathcal{A}}_{\mathbb{M}}(S)
$$

Next we specify some conditions on the sequence $\mathbb{M}$ that will have important consequences on the previous classes or spaces.

Definition 2.5 We say:

(i) $\mathbb{M}$ is logarithmically convex [for short, (lc)] if

$$
M_{p}^{2} \leq M_{p-1} M_{p+1}, \quad p \in \mathbb{N}
$$

(ii) $\mathbb{M}$ is derivation closed [for short, (dc)] if there exists $A>0$ such that

$$
M_{p+1} \leq A^{p+1} M_{p}, \quad p \in \mathbb{N}_{0}
$$

(iii) $\mathbb{M}$ is of moderate growth [briefly, (mg)] if there exists $B>0$ such that

$$
M_{p+q} \leq B^{p+q} M_{p} M_{q}, \quad p, q \in \mathbb{N}_{0} .
$$

(iv) $\mathbb{M}$ satisfies the strong non-quasianalyticity condition [for short, (snq)] if there exists $C>0$ such that

$$
\sum_{q \geq p} \frac{M_{q}}{(q+1) M_{q+1}} \leq C \frac{M_{p}}{M_{p+1}}, \quad p \in \mathbb{N}_{0} .
$$

Obviously, (mg) implies (dc).

Definition 2.6 (Thilliez [20]) We say $\mathbb{M}$ is strongly regular if it verifies (lc), (mg), and (snq).

Definition 2.7 For a sequence $\mathbb{M}$, we define the sequence of quotients $\mathbf{m}=\left(m_{p}\right)_{p \in \mathbb{N}_{0}}$ by

$$
m_{p}:=\frac{M_{p+1}}{M_{p}}, \quad p \in \mathbb{N}_{0} .
$$

It is obvious that $\mathbb{M}$ is (lc) if, and only if, $\mathbf{m}$ is nondecreasing.

Definition 2.8 Let $\mathbb{M}$ and $\mathbb{L}$ be sequences, we say that $\mathbb{M}$ is equivalent to $\mathbb{L}$, and we write $\mathbb{M} \approx \mathbb{L}$, if there exist positive constants $A, B>0$ such that

$$
A^{p} L_{p} \leq M_{p} \leq B^{p} L_{p}, \quad p \in \mathbb{N}_{0}
$$


Example 2.9 We mention some interesting examples. In particular, those in (i) and (iii) appear in the applications of summability theory to the study of formal power series solutions for different kinds of equations.

(i) The sequences $\mathbb{M}_{\alpha, \beta}:=\left(p !^{\alpha} \prod_{m=0}^{p} \log ^{\beta}(e+m)\right)_{p \in \mathbb{N}_{0}}$, where $\alpha>0$ and $\beta \in \mathbb{R}$, are strongly regular (more precisely, in case $\beta<0$ the sequence is equivalent to a strongly regular one, see Remark 2.10). For $\beta=0$, we have the best-known example of strongly regular sequence, $\mathbb{M}_{\alpha, 0}=\left(p !^{\alpha}\right)_{p \in \mathbb{N}_{0}}$, called the Gevrey sequence of order $\alpha$.

(ii) The sequence $\mathbb{M}_{0, \beta}:=\left(\prod_{m=0}^{p} \log ^{\beta}(e+m)\right)_{p \in \mathbb{N}_{0}}$, with $\beta>0$, is (lc), (mg) and $\mathbf{m}$ tends to infinity, but (snq) is not satisfied.

(iii) For $q>1, \mathbb{M}_{q}:=\left(q^{p^{2}}\right)_{p \in \mathbb{N}_{0}}$ is (lc) and (snq), but not (mg).

Remark 2.10 For any sequence $\mathbb{M}$, the classes $\mathcal{A}_{\mathbb{M}}(S), \tilde{\mathcal{A}}_{\mathbb{M}}^{\mathrm{u}}(S)$, and $\tilde{\mathcal{A}}_{\mathbb{M}}(S)$ are vector spaces. If $\mathbb{M}$ is (lc), they are algebras; if $\mathbb{M}$ is (dc), they are stable under taking derivatives. Moreover, equivalent sequences define the same classes.

Definition 2.11 Let $f$ be a function defined in a sectorial region $G=G(d, \gamma)$, and $\theta$ be a direction in $G$, i.e. $|\theta-d|<\pi \gamma / 2$. We say $f$ has $\mathbb{M}$-asymptotic expansion $\hat{f}=\sum_{n=0}^{\infty} a_{n} z^{n}$ in direction $\theta$ if there exist $r_{\theta}, C_{\theta}, A_{\theta}>0$ such that the segment $\left(0, r_{\theta} \mathrm{e}^{i \theta}\right]$ is contained in $G$, and for every $z \in\left(0, r_{\theta} \mathrm{e}^{i \theta}\right]$ and every $p \in \mathbb{N}_{0}$ one has

$$
\left|f(z)-\sum_{n=0}^{p-1} a_{n} z^{n}\right| \leq C_{\theta} A_{\theta}^{p} M_{p}|z|^{p}
$$

In this case, we say the type is $1 / A_{\theta}$. Of course, the definition makes sense as long as the function is defined only in direction $\theta$ near the origin, i.e. in a segment $\left(0, r \mathrm{e}^{i \theta}\right]$ for suitable $r>0$.

One may accordingly define classes of formal power series

$$
\mathbb{C}[[z]]_{\mathbb{M}, \mathrm{A}}=\left\{\hat{f}=\sum_{n=0}^{\infty} a_{n} z^{n} \in \mathbb{C}[[z]]:|\hat{f}|_{\mathbb{M}, \mathrm{A}}:=\sup _{p \in \mathbb{N}_{0}} \frac{\left|a_{p}\right|}{A^{p} M_{p}}<\infty\right\} .
$$

$\left(\mathbb{C}[[z]]_{\mathbb{M}, \mathrm{A}},||_{\mathbb{M}, \mathrm{A}}\right)$ is a Banach space, and we put $\mathbb{C}[[z]]_{\mathbb{M}}:=\cup_{A>0} \mathbb{C}[[z]]_{\mathbb{M}, \mathrm{A}}$.

Remark 2.12 Given $f \in \tilde{\mathcal{A}}_{\mathbb{M}}(G)$ with $f \sim_{\mathbb{M}} \hat{f}=\sum_{p=0}^{\infty} a_{p} z^{p}$, it is plain to check that for every bounded proper subsector $T$ of $G$ and every $p \in \mathbb{N}_{0}$, one has

$$
a_{p}=\lim _{\substack{z \rightarrow 0 \\ z \in T}} \frac{f^{(p)}(z)}{p !}
$$

and we can set $f^{(p)}(0):=p ! a_{p}$. Moreover, if we define $\tilde{\mathcal{B}}(f):=\hat{f}$, it is straightfor-

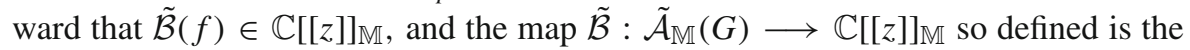
asymptotic Borel map. If $S$ is a sector, using Proposition 2.4 we see that the asymptotic Borel map is also well defined on $\mathcal{A}_{\mathbb{M}}(S)$ and $\tilde{\mathcal{A}}_{\mathbb{M}}^{\mathrm{u}}(S)$. 


\subsection{Classical Quasianalyticity Results}

We introduce first the notions of flatness and quasianalyticity.

Definition 2.13 A function $f$ in any of the previous classes is said to be $f$ lat if $\tilde{\mathcal{B}}(f)$ is the null formal power series (denoted $\hat{0}$ ), or in other words, $f \sim_{\mathbb{M}} \hat{0}$.

Definition 2.14 Let $S$ be a sector, $G$ a sectorial region and $\mathbb{M}=\left(M_{p}\right)_{p \in \mathbb{N}_{0}}$ be a sequence of positive numbers. We say that $\mathcal{A}_{\mathbb{M}}(S), \tilde{\mathcal{A}}_{\mathbb{M}}^{\mathrm{u}}(S)$, or $\tilde{\mathcal{A}}_{\mathbb{M}}(G)$ is quasianalytic if it does not contain nontrivial flat functions (in other words, the Borel map is injective in this class).

In order to simplify some statements or to avoid trivial situations, from now on in this paper, we will assume the standard property that

The sequence $\mathbb{M}$ is logarithmically convex with $\lim _{p \rightarrow \infty} m_{p}=\infty$.

The following result characterizes quasianalyticity for the classes of functions with uniformly bounded derivatives in an unbounded sector. It first appeared in RodríguezSalinas [17], although it is frequently attributed to Korenbljum [10].

Theorem 2.15 ([17], Theorem 12)Let $\mathbb{M}$ and $\gamma>0$ be given. The following statements are equivalent:

(i) The class $\mathcal{A}_{\mathbb{M}}\left(S_{\gamma}\right)$ is quasianalytic.

(ii) $\sum_{p=0}^{\infty}\left(\frac{1}{(p+1) m_{p}}\right)^{1 /(\gamma+1)}$ diverges.

This result can be rewritten in terms of the classical notion of exponent of convergence of a sequence.

Proposition 2.16 ([6], p. 65) Let $\left(c_{n}\right)_{n \in \mathbb{N}_{0}}$ be a nondecreasing sequence of positive real numbers tending to infinity. The exponent of convergence of $\left(c_{n}\right)_{n}$ is defined as

$$
\lambda_{\left(c_{n}\right)}:=\inf \left\{\mu>0: \sum_{n=0}^{\infty} \frac{1}{c_{n}^{\mu}} \text { converges }\right\}
$$

(if the previous set is empty, we put $\lambda_{\left(c_{n}\right)}=\infty$ ). Then, one has

$$
\lambda_{\left(c_{n}\right)}=\limsup _{n \rightarrow \infty} \frac{\log (n)}{\log \left(c_{n}\right)}
$$

According to this last formula, we may define the index

$$
\omega(\mathbb{M}):=\liminf _{p \rightarrow \infty} \frac{\log \left(m_{p}\right)}{\log (p)}
$$


in such a way that

$$
\lambda_{\left(m_{p}\right)}=\frac{1}{\omega(\mathbb{M})}, \quad \lambda_{\left((p+1) m_{p}\right)}=\frac{1}{\omega(\mathbb{M})+1} .
$$

So, Theorem 2.15 may be stated as

Corollary 2.17 Let $\mathbb{M}$ and $\gamma>0$ be given. The following statements are equivalent:

(i) The class $\mathcal{A}_{\mathbb{M}}\left(S_{\gamma}\right)$ is quasianalytic.

(ii) $\gamma>\omega(\mathbb{M})$, or $\gamma=\omega(\mathbb{M})$ and $\sum_{p=0}^{\infty}\left(\frac{1}{(p+1) m_{p}}\right)^{1 /(\omega(\mathbb{M})+1)}$ diverges.

Remark 2.18 The problem of quasianalyticity for classes of functions with uniformly bounded derivatives in bounded regions has also been treated. In the works of Trunov and Yulmukhametov [23,25], a characterization is given, for a convex bounded region containing 0 in its boundary, in terms of the sequence $\mathbb{M}$ and of the way the boundary approaches 0 . In particular, for bounded sectors, if $\gamma \leq 1, d \in \mathbb{R}$ and $r>0$, it turns out that the class $\mathcal{A}_{\mathbb{M}}(S(d, \gamma, r))$ is quasianalytic precisely when condition (ii) above is satisfied.

The study of quasianalyticity for the classes of functions with uniform $\mathbb{M}$ asymptotic expansion in an unbounded sector rests on the following statement by Mandelbrojt.

Theorem 2.19 ([16], Sect. 2.4.III) Let $\mathbb{M}$ be given, $H=\{z \in \mathbb{C}: \Re(z)>0\}$ and $\gamma>0$. The following statements are equivalent:

(i) If $f \in \mathcal{H}(H)$ and there exist $A, C>0$ such that

$$
|f(z)| \leq \frac{C A^{p} M_{p}}{|z|^{\gamma p}}, \quad z \in H, \quad p \in \mathbb{N}_{0}
$$

then $f$ identically vanishes.

(ii) $\sum_{p=0}^{\infty}\left(\frac{1}{m_{p}}\right)^{1 / \gamma}$ diverges.

Observe that a function $f$ is holomorphic in $H$ and verifies the estimates (2.2) if, and only if, the function $g$ given by $g(z):=f\left(1 / z^{1 / \gamma}\right)$ belongs to $\tilde{\mathcal{A}}_{\mathbb{M}}^{\mathrm{u}}\left(S_{\gamma}\right)$ and is flat. From this fact and the first equality in (2.1), it is immediate to deduce the next characterization.

Corollary 2.20 (Generalized Watson's lemma for uniform asymptotics) Let $\mathbb{M}$ and $\gamma>0$ be given. The following are equivalent:

(i) $\tilde{\mathcal{A}}_{\mathbb{M}}^{\mathrm{u}}\left(S_{\gamma}\right)$ is quasianalytic.

(ii) $\sum_{p=0}^{\infty}\left(\frac{1}{m_{p}}\right)^{1 / \gamma}$ diverges.

(iii) $\gamma>\omega(\mathbb{M})$, or $\gamma=\omega(\mathbb{M})$ and $\sum_{p=0}^{\infty}\left(\frac{1}{m_{p}}\right)^{1 / \omega(\mathbb{M})}$ diverges. 
Remark 2.21 This theorem holds true for bounded sectors $S(0, \gamma, r)$ with similar arguments. Proceeding as in [8, Theorem 2.19], we only need to modify the proof of (ii) $\Rightarrow$ (i) by considering the transformation $w(z)=1 /\left(z+(1 / r)^{1 / \gamma}\right)^{\gamma}$, which maps $H$ into a region $D$ contained in $S(0, \gamma, r)$ : given a flat function $g \in \tilde{\mathcal{A}}_{\mathbb{M}}^{\mathrm{u}}(S(0, \gamma, r))$, the function $f(w):=g(z(w))$ is defined in $H$ and, by Mandelbrojt's theorem, it identically vanishes.

Regarding the class of functions with (non-uniform) asymptotic expansion in a sectorial region $G$, we first express flatness in $\tilde{\mathcal{A}}_{\mathbb{M}}(G)$ by means of an auxiliary function: for $t>0$, we define

$$
M(t):=\sup _{p \in \mathbb{N}_{0}} \log \left(\frac{t^{p}}{M_{p}}\right)= \begin{cases}p \log t-\log \left(M_{p}\right) & \text { if } t \in\left[m_{p-1}, m_{p}\right), p=1,2, \ldots, \\ 0 & \text { if } t \in\left[0, m_{0}\right),\end{cases}
$$

which is a non-decreasing continuous map in $[0, \infty)$ with $\lim _{t \rightarrow \infty} M(t)=\infty$. Then, we have the following result.

Theorem 2.22 ([21], Proposition 4) Given $f \in \mathcal{H}(G)$, the following are equivalent:

(i) $f \in \tilde{\mathcal{A}}_{\mathbb{M}}(G)$ and $f$ is flat.

(ii) For every bounded proper subsector $T$ of $G$, there exist $c_{1}, c_{2}>0$ with

$$
|f(z)| \leq c_{1} \mathrm{e}^{-M\left(1 /\left(c_{2}|z|\right)\right)}, \quad z \in T .
$$

Remark 2.23 In the conditions of Definition 2.11, if $\hat{f}$ is the null series, then we say that $f$ is $\mathbb{M}$-flat in direction $\theta$. As in the previous statement, this amounts to the existence of $r_{\theta}, C_{\theta}, A_{\theta}>0$ such that the segment $\left(0, r_{\theta} \mathrm{e}^{i \theta}\right]$ is contained in $G$, and for every $z \in\left(0, r_{\theta} \mathrm{e}^{i \theta}\right]$ one has

$$
|f(z)| \leq C_{\theta} \mathrm{e}^{-M\left(1 /\left(A_{\theta}|z|\right)\right)} .
$$

Suppose moreover that $f$ is bounded throughout the (bounded or not) sectorial region $G$. Since the function $t \mapsto \mathrm{e}^{-M(t)}$ is non-increasing in $[0, \infty)$, it is obvious that $f$ is M-flat in direction $\theta$ if, and only if, there exist $\tilde{C}_{\theta}>0$ and the same constant $A_{\theta}>0$ as before, such that for every $z \in G$ with $\arg z=\theta$ one has

$$
|f(z)| \leq \tilde{C}_{\theta} \mathrm{e}^{-M\left(1 /\left(A_{\theta}|z|\right)\right)} .
$$

This fact will be used later on.

\subsection{Quasianalyticity Results via Proximate Orders}

An easy characterization of quasianalyticity in the classes $\tilde{\mathcal{A}}_{\mathbb{M}}(G)$ may be given thanks to the notion of proximate order, appearing in the theory of growth of entire functions and developed, initially, by Lindelöf and Valiron. We will focus our discussion mainly on the results given by Maergoiz (see [13]).

Definition 2.24 We say a real function $\rho$, defined on $(c, \infty)$ for some $c \geq 0$, is a proximate order, if the following hold: 
(A) $\rho$ is continuous and piecewise continuously differentiable in $(c, \infty)$ (meaning that it is differentiable except possibly at a sequence of points, tending to infinity, at any of which it is continuous and has distinct finite lateral derivatives),

(B) $\rho(r) \geq 0$ for every $r>c$,

(C) $\lim _{r \rightarrow \infty} \rho(r)=\rho_{\infty}<\infty$,

(D) $\lim _{r \rightarrow \infty} r \rho^{\prime}(r) \ln r=0$.

In case the value $\rho_{\infty}$ in (C) is positive (respectively, is 0 ), we say $\rho$ is a nonzero (resp. zero) proximate order.

Remark 2.25 If $\rho$ is a proximate order with limit $\rho_{\infty}$ at infinity and $\alpha>\rho_{\infty}$, then there exists $r(\alpha)>1$ such that $\rho(r)<\alpha$ for $r>r(\alpha)$ and, consequently,

$$
r^{\rho(r)}<r^{\alpha}, \quad r>r(\alpha) .
$$

We now associate to a nonzero proximate order a class of functions with nice properties, which will play a prominent role in our Phragmén-Lindelöf result.

Theorem 2.26 ([13], Theorem 2.4) Let $\rho$ be a nonzero proximate order such that $\lim _{r \rightarrow \infty} \rho(r)=\rho_{\infty}$. For every $\gamma>0$, there exists an analytic function $V$ in $S_{\gamma}$ such that:

(I) For every $z \in S_{\gamma}$,

$$
\lim _{r \rightarrow \infty} \frac{V(z r)}{V(r)}=z^{\rho_{\infty}},
$$

uniformly in the compact sets of $S_{\gamma}$.

(II) $\overline{V(z)}=V(\bar{z})$ for every $z \in S_{\gamma}$ (where, for $z=(|z|, \arg z$ ), we put $\bar{z}=$ $(|z|,-\arg z))$.

(III) $V$ is positive in $(0, \infty)$, monotone increasing and $\lim _{r \rightarrow 0} V(r)=0$.

(IV) The function $t \mapsto V\left(\mathrm{e}^{t}\right)$ is strictly convex in $\mathbb{R}$ (i.e. $V$ is strictly convex relative to $\log (r))$.

(V) The function $r \mapsto \log (V(r))$ is strictly concave in $(0, \infty)$.

(VI) The function $r \mapsto \rho_{V}(r):=\log (V(r)) / \log (r), r>0$, is a proximate order equivalent to $\rho$, i.e.,

$$
\lim _{r \rightarrow \infty} V(r) / r^{\rho(r)}=\lim _{r \rightarrow \infty} r^{\rho_{V}(r)} / r^{\rho(r)}=1 .
$$

Given $\gamma>0$ and $\rho$ as before, $\operatorname{MF}(\gamma, \rho)$, will denote the set of Maergoiz functions $V$ defined in $S_{\gamma}$ and satisfying the conditions (I)-(VI) of Theorem 2.26.

Before returning to the study of quasianalyticity, we indicate how to go from sequences to proximate orders (for more information on this relation and its reversion, see [9]). Given $\mathbb{M}$ and its associated function $M$, for $t$ large enough, we can consider

$$
d_{\mathbb{M}}(t):=\log (M(t)) / \log (t)
$$

The following result characterizes those sequences for which $d_{\mathbb{M}}$ is a proximate order. 
Theorem 2.27 ([9], Theorem 3.6) Let $\mathbb{M}$ be given. The following are equivalent:

(a) $d_{\mathbb{M}}$ is a proximate order with $\lim _{t \rightarrow \infty} d_{\mathbb{M}}(t) \in(0, \infty)$.

(b) There exists $\lim _{p \rightarrow \infty} \log \left(m_{p} / M_{p}^{1 / p}\right) \in(0, \infty)$.

(c) $\boldsymbol{m}$ is regularly varying with a positive index of regular variation.

(d) There exists $\omega>0$ such that for every natural number $\ell \geq 2$,

$$
\lim _{p \rightarrow \infty} \frac{m_{\ell p}}{m_{p}}=\ell^{\omega}
$$

In case any of these statements holds, the value of the limit mentioned in $(b)$, that of the index mentioned in $(c)$, and that of the constant $\omega$ in $(d)$ is $\omega(\mathbb{M})$, and the limit in (a) is $1 / \omega(\mathbb{M})$.

A less restrictive condition on the sequence $\mathbb{M}$, namely the admissibility of a proximate order, is indeed sufficient for our purposes.

Theorem 2.28 ([9], Theorem 4.14) Given $\mathbb{M}$, the following conditions are equivalent:

(e) There exists a (lc) sequence $\mathbb{L}$, with quotients tending to infinity, such that $\mathbb{L} \approx \mathbb{M}$ and $d_{\mathbb{L}}$ is a nonzero proximate order.

(f) $\mathbb{M}$ admits a nonzero proximate order, i.e., there exist a nonzero proximate order $\rho$ and constants $A$ and $B$ such that

$$
A \leq \log (t)\left(\rho(t)-d_{\mathbb{M}}(t)\right) \leq B, \quad t \text { large enough }
$$

From this result, we deduce that whenever a class $\tilde{\mathcal{A}}_{\mathbb{M}}(G)\left(\right.$ or $\tilde{\mathcal{A}}_{\mathbb{M}}^{\mathrm{u}}(S)$ or $\mathcal{A}_{\mathbb{M}}(S)$ ) is defined in terms of a sequence $\mathbb{M}$ admitting a nonzero proximate order, we can exchange $\mathbb{M}$ by another equivalent (lc) sequence $\mathbb{L}$, whose sequence of quotients is regularly varying. Then, we can briefly say that the $\mathbb{M}$-asymptotic expansion of a function $f \in \tilde{\mathcal{A}}_{\mathbb{M}}(G)=\tilde{\mathcal{A}}_{\mathbb{L}}(G)$ has log-convex regularly varying constraints.

Remark 2.29 If $\mathbb{M}$ admits a nonzero proximate order $\rho$, it is clear that $\lim _{t \rightarrow \infty} d_{\mathbb{M}}(t)=$ $\lim _{t \rightarrow \infty} \rho(t)$ [see (2.4)], and from [9, Remark 4.15], we deduce that this common value is $1 / \omega(\mathbb{M})$.

Remark 2.30 If $\mathbb{M}$ admits a nonzero proximate order $\rho$, then for every $\gamma>0$, we know that for the function $V \in \operatorname{MF}(\gamma, \rho)$ given by Theorem 2.26 there exist positive constants $A, B, t_{0}$ such that

$$
A V(t) \leq M(t) \leq B V(t), \quad t>t_{0}
$$

Example 2.31 We provide an example showing that the results in this paper are indeed generalizations of the ones by Fruchard and Zhang [3]. Consider, for $\alpha>0$ and $\beta \neq 0$, the sequence $\mathbb{M}_{\alpha, \beta}$ introduced in Example 2.9(i). It is not equivalent to any Gevrey sequence and, as indicated in [9, Remark 4.2], it admits the nonzero proximate order $\rho_{\alpha, \beta}$ given by

$$
\rho_{\alpha, \beta}(t)=\frac{1}{\alpha}-\frac{\beta}{\alpha} \frac{\log (\log (t))}{\log (t)}, \quad t \text { large enough. }
$$


In [9, Remark 4.15], it has been shown that sequences admitting a nonzero proximate order are indeed strongly regular. So, as indicated in [18, Remark 4.11(iii)], for such sequences $\mathbb{M}$ one may construct nontrivial flat functions in $\tilde{\mathcal{A}}_{\mathbb{M}}\left(G_{\omega(\mathbb{M})}\right)$, what allows us to state the following version of Watson's Lemma for non-uniform asymptotics.

Theorem 2.32 ([18], Corollary 4.12) Suppose $\mathbb{M}$ admits a nonzero proximate order, and let $\gamma>0$ be given. The following statements are equivalent:

(i) $\tilde{\mathcal{A}}_{\mathbb{M}}\left(G_{\gamma}\right)$ is quasianalytic.

(ii) $\gamma>\omega(\mathbb{M})$.

Moreover, for such sequences, we can generalize Borel-Ritt-Gevrey theorem [18] and the Gevrey summability theory following Balser's moment summability methods, see [11].

Remark 2.33 Corollaries 2.17, 2.20 and Theorem 2.32 are also valid if we change the bisecting direction of the considered sectorial region.

\section{M-Flatness Extension}

From this point onward, we will assume not only that the sequence $\mathbb{M}$ is logarithmically convex with $\lim _{p \rightarrow \infty} m_{p}=\infty$, but also that

\section{The sequence $\mathbb{M}$ admits a nonzero proximate order.}

This is not a strong assumption for strongly regular sequences, since it is satisfied by every such sequence appearing in applications (the Gevrey ones, or the one associated to the $1^{+}$-level asymptotics). However, note that there are strongly regular sequences which do not satisfy it, see [9].

We are ready for proving an important lemma about the extension of $\mathbb{M}$-flatness from a boundary direction into a whole small sector for functions bounded there and admitting a continuous extension to the boundary (considered in $\mathcal{R}$, i.e., disregarding the origin). First, we recall a classical version of Phragmén-Lindelöf theorem needed in the proof.

Theorem 3.1 (Phragmén-Lindelöf Theorem, [22], p. 177) Let $f$ be a function holomorphic in a sector $S=S(d, \gamma, \rho)$, continuous and bounded by $C$ in the boundary $\partial S$. Suppose there exist $K, L>0$ and $\omega>\gamma$ such that

$$
|f(z)|<K \mathrm{e}^{L|z|^{-1 / \omega}}
$$

for every $z \in S$. Then $f$ is bounded by $C$ in the sector $S$.

Now we obtain an analog of Phragmén-Lindelöf theorem for $\mathbb{M}$-flat functions in a sector.

Lemma 3.2 Let $\mathbb{M}$ and $0<\gamma<\omega(\mathbb{M})$ be given. Suppose $f$ is a bounded holomorphic function in $S_{\gamma}$ that admits a continuous extension to the boundary $\partial S_{\gamma}$, and that is 
$\mathbb{M}$-flat in direction $d=\pi \gamma / 2$. Then for every $0<\delta<\pi \gamma$, there exist constants $k_{1}(\delta), k_{2}(\delta)>0$ with

$$
|f(z)| \leq k_{1} \mathrm{e}^{-M\left(1 /\left(k_{2}|z|\right)\right)}, \quad \arg z \in[-\pi \gamma / 2+\delta, \pi \gamma / 2] .
$$

Proof For simplicity, we denote $\omega:=\omega(\mathbb{M})$. We fix $0<\delta<\pi \gamma$. Since $\gamma<\omega$, we have that

$$
\begin{aligned}
& \frac{\pi}{2}<\beta=\beta(\delta):=\frac{1}{\omega}\left(\frac{\pi}{2} \omega+\frac{\delta}{2}\right)<\pi, \\
& -\frac{\pi}{2}+\frac{\delta}{2 \omega}<\alpha=\alpha(\delta):=\frac{1}{\omega}\left(\frac{\pi}{2} \omega-\pi \gamma+\frac{\delta}{2}\right)<\frac{\pi}{2} .
\end{aligned}
$$

Then we take $\varepsilon, \eta>0$ (depending on $\delta$ ) such that

$$
\cos \beta+\varepsilon \leq-\eta<0
$$

Since $\mathbb{M}$ admits a nonzero proximate order $\rho$, by Theorem 2.26 there exist $V \in$ $\operatorname{MF}(2 \omega, \rho)$ and positive constants $A, B, t_{0}$ such that (2.5) holds (see Remark 2.30).

According to Remark 2.23, and specifically to (2.3), there exist $c_{1}, c_{2}>0$ with

$$
|f(z)| \leq c_{1} \mathrm{e}^{-M\left(1 /\left(c_{2}|z|\right)\right)}, \quad \arg z=\pi \gamma / 2
$$

Choose $d_{2}>0$ such that $c_{2}^{-1 / \omega}>d_{2}$, and take $a \in \mathcal{R}$ with

$$
\arg a=\frac{\omega \pi}{2}-\frac{\pi \gamma}{2}+\frac{\delta}{2}, \quad 0<|a|<\left(\frac{A d_{2}}{2}\right)^{\omega} .
$$

It is clear that $\varepsilon<1$, so we have that

$$
\cos \left(\frac{\arg a-\arg z}{\omega}\right)+\varepsilon \leq 2
$$

for every $z \in \overline{S_{\gamma}}$.

We observe that $\arg a / z \in[\omega \alpha, \omega \beta] \subseteq(-\pi \omega / 2, \pi \omega)$ for every $z \in \overline{S_{\gamma}}$. Taking into account Remark 2.29 and using property (I) of the functions in $\operatorname{MF}(2 \omega, \rho)$ we see that

$$
\lim _{|z| \rightarrow 0} \frac{V(a / z)}{|a|^{1 / \omega} V(1 /|z|)}=\mathrm{e}^{i(\arg a-\arg z) / \omega}
$$

uniformly for $\arg z \in[-\pi \gamma / 2, \pi \gamma / 2]$. Consequently,

$$
\lim _{|z| \rightarrow 0} \Re\left(\frac{V(a / z)}{|a|^{1 / \omega} V(1 /|z|)}\right)=\cos ((\arg a-\arg z) / \omega)
$$


uniformly for $\arg z \in[-\pi \gamma / 2, \pi \gamma / 2]$, and we deduce that

$$
\begin{aligned}
& |a|^{1 / \omega} V\left(\frac{1}{|z|}\right)(\cos ((\arg a-\arg z) / \omega)-\varepsilon) \leq \Re\left(V\left(\frac{a}{z}\right)\right), \\
& |a|^{1 / \omega} V\left(\frac{1}{|z|}\right)(\cos ((\arg a-\arg z) / \omega)+\varepsilon) \geq \Re\left(V\left(\frac{a}{z}\right)\right),
\end{aligned}
$$

for $|z|<s_{1}$ small enough and $\arg z \in[-\pi \gamma / 2, \pi \gamma / 2]$. For convenience, we choose $s_{1}<1 /\left(t_{0} c_{2}\right)$. Consider the function

$$
F(z):=f(z) \mathrm{e}^{V(a / z)} .
$$

The function $z \mapsto V(a / z)$ is holomorphic in $S(\arg a, 2 \omega) \supset \overline{S_{\gamma}}$, so $F$ is holomorphic in $S_{\gamma}$ and continuous up to $\partial S_{\gamma}$. Our aim is to apply the Phragmén-Lindelöf Theorem 3.1 to this function in a suitable bounded sector $S\left(0, \gamma, s_{3}\right)$.

If $\arg z=-\pi \gamma / 2$, we have that $\arg a-\arg z=\beta \omega$. Then, since $f$ is bounded in $\overline{S_{\gamma}}$ by a constant $K>0$, by using (3.4), we see that for $|z|<s_{1}$,

$$
|F(z)| \leq K \mathrm{e}^{\Re(V(a / z))} \leq K \mathrm{e}^{(\cos \beta+\varepsilon)|a|^{1 / \omega} V(1 /|z|)} \leq K \mathrm{e}^{-\eta|a|^{1 / \omega} V(1 /|z|)} .
$$

Now, observe that $V(1 /|z|)>0$ [property (III)], so we deduce that $|F(z)| \leq K$ for every $z$ with $|z|<s_{1}$ and $\arg z=-\pi \gamma / 2$.

If $\arg z=\pi \gamma / 2$, we have that $\arg a-\arg z=\alpha \omega$. Then, from (3.1), (2.5), (3.2) and (3.4), we see that, if $|z|<s_{1}$,

$$
|F(z)| \leq c_{1} \mathrm{e}^{-M\left(1 /\left(c_{2}|z|\right)\right)} \mathrm{e}^{(\cos \alpha+\varepsilon)|a|^{1 / \omega} V(1 /|z|)} \leq c_{1} \mathrm{e}^{-A V\left(1 /\left(c_{2}|z|\right)\right)+2|a|^{1 / \omega} V(1 /|z|)} .
$$

Using property (I) of the functions in $\operatorname{MF}(2 \omega, \rho)$ we have that

$$
\lim _{|z| \rightarrow 0} \frac{V\left(1 /\left(c_{2}|z|\right)\right)}{V(1 /|z|)}=c_{2}^{-1 / \omega}
$$

Then, for $|z|<s_{2} \leq s_{1}$ small enough, we have that $V\left(1 /\left(c_{2}|z|\right)\right) \geq d_{2} V(1 /|z|)$, and we conclude that

$$
|F(z)| \leq c_{1} \mathrm{e}^{\left(-A d_{2}+2|a|^{1 / \omega}\right) V(1 /|z|)}, \quad|z|<s_{2}, \quad \arg z=\pi \gamma / 2 .
$$

Since $|a|$ has been chosen small enough in order that $-A d_{2}+2|a|^{1 / \omega}<0$, we deduce that $|F(z)| \leq c_{1}$ for every $|z|<s_{2}$ and $\arg z=\pi \gamma / 2$.

For $z \in S_{\gamma}$ with $|z|<s_{1}$, by using (3.2) and (3.4), we have that

$$
\Re\left(V\left(\frac{a}{z}\right)\right) \leq 2|a|^{1 / \omega} V\left(\frac{1}{|z|}\right) .
$$

As $\gamma<\omega$, there exists $\mu>0$ such that $\gamma<\mu<\omega$. By property (VI), we know that $\rho_{V}: t \mapsto \log (V(t)) / \log (t)$ is a proximate order equivalent to $\rho$, hence tending 
to $1 / \omega$ at infinity. Then, we can apply Remark 2.25 : there exists $0<s_{3} \leq s_{2}$ small enough such that for every $z \in S_{\gamma},|z| \leq s_{3}$,

$$
\Re\left(V\left(\frac{a}{z}\right)\right) \leq 2|a|^{1 / \omega}\left(\frac{1}{|z|}\right)^{1 / \mu} .
$$

Since $f$ is bounded in $S_{\gamma}$, we have that

$$
|F(z)| \leq K \exp \left(2|a|^{1 / \omega}|z|^{-1 / \mu}\right), \quad z \in S_{\gamma}, \quad|z| \leq s_{3},
$$

and, in particular,

$$
|F(z)| \leq K \exp \left(2|a|^{1 / \omega} s_{3}^{-1 / \mu}\right), \quad z \in S_{\gamma}, \quad|z|=s_{3}
$$

By applying Phragmén-Lindelöf Theorem 3.1 to the function $F$ in $S\left(0, \gamma, s_{3}\right)$, we obtain that

$$
|F(z)| \leq K_{0}:=\max \left(K, c_{1}, K \exp \left(2|a|^{1 / \omega} s_{3}^{-1 / \mu}\right)\right)
$$

for $|z| \leq s_{3}$ and $\arg z \in[-\pi \gamma / 2, \pi \gamma / 2]$.

Consequently, using (3.3), if $|z| \leq s_{3}$ and $\arg z \in[-\pi \gamma / 2, \pi \gamma / 2]$, we have that

$$
|f(z)| \leq K_{0} \mathrm{e}^{\Re(-V(a / z))} \leq K_{0} \mathrm{e}^{-(\cos ((\arg a-\arg z) / \omega)-\varepsilon)|a|^{1 / \omega} V(1 /|z|)} .
$$

Assuming that $\arg z \in[-\pi \gamma / 2+\delta, \pi \gamma / 2]$, we deduce that

$$
\cos ((\arg a-\arg z) / \omega) \geq \cos \left(\frac{\pi}{2}-\frac{\delta}{2 \omega}\right)=-\cos \beta \geq \eta+\varepsilon>0 .
$$

Then, for $r_{2}:=\eta|a|^{1 / \omega}>0$, we find that for every $z$ with $\arg z \in[-\pi \gamma / 2+\delta, \pi \gamma / 2]$ and $|z|<s_{3}$, we have that

$$
|f(z)| \leq K_{0} \mathrm{e}^{-r_{2} V(1 /|z|)}
$$

Choose $k_{2}>0$ such that $\left(1 / k_{2}\right)^{1 / \omega}<r_{2} / B$. Property (I) of the functions in $\operatorname{MF}(2 \omega, \rho)$ implies that, for $z$ with $|z|<s_{4}<\min \left(s_{3}, 1 /\left(t_{0} k_{2}\right)\right)$, small enough, and $\arg z \in$ $[-\pi \gamma / 2+\delta, \pi \gamma / 2]$, we have

$$
|f(z)| \leq K_{0} \mathrm{e}^{-B V\left(1 /\left(k_{2}|z|\right)\right)} \leq K_{0} \mathrm{e}^{-M\left(1 /\left(k_{2}|z|\right)\right)} .
$$

We take $k_{1}:=K_{0} \mathrm{e}^{M\left(1 /\left(k_{2} s_{4}\right)\right)} \geq K_{0}$. Then, since $M$ is nondecreasing, if $|z| \geq s_{4}$ and $\arg z \in[-\pi \gamma / 2+\delta, \pi \gamma / 2]$, we have

$$
|f(z)| \leq K \leq K_{0}=k_{1} \mathrm{e}^{-M\left(1 /\left(k_{2} s_{4}\right)\right)} \leq k_{1} \mathrm{e}^{-M\left(1 /\left(k_{2}|z|\right)\right)},
$$


which concludes the proof.

Remark 3.3 Some comments are in order concerning the statement or proof of the previous result.

By a simple rotation, one may easily check that the validity of Lemma 3.2 does not depend on the bisecting direction of the sector where the function $f$ is defined. Moreover, one could slightly weaken the hypotheses by considering a function $f$ holomorphic in $S_{\gamma}$ that admits a continuous extension to the direction $d=\pi \gamma / 2$, in which it is $\mathbb{M}$-flat, and that is bounded in every (half-open) sector

$$
\left\{z \in \mathcal{R}: \arg z \in\left(-\frac{\pi \gamma}{2}+\delta, \frac{\pi \gamma}{2}\right]\right\}, \quad \delta>0
$$

Indeed, we may give a more precise information about the type. Following the previous proof, one notes that

$$
\begin{aligned}
k_{2} & =k_{2}(\delta)>\left(\frac{B}{r_{2}}\right)^{\omega}=\left(\frac{B}{\eta|a|^{1 / \omega}}\right)^{\omega} \geq\left(\frac{2 B}{A d_{2} \cos \left(\frac{\pi}{2}-\frac{\delta}{2 \omega}\right)}\right)^{\omega} \\
& \geq\left(\frac{2 B}{A}\right)^{\omega}\left(\frac{1}{\sin \left(\frac{\delta}{2 \omega}\right)}\right)^{\omega} c_{2},
\end{aligned}
$$

and $k_{2}$ may be made arbitrarily close to the last expression at the price of enlarging the constant $k_{1}=k_{1}(\delta)$. So, the original type $c_{2}$ is basically affected by a precise factor when moving to a direction $\theta=-\pi \gamma / 2+\delta$ with $0<\delta<\pi \gamma$. It is obvious that $k_{2}(\delta)$ explodes at least like $1 / \sin ^{\omega} \delta$ as $\delta \rightarrow 0$. This means that the type of the null asymptotic expansion tends to 0 as the direction in the sector approaches the boundary $d=-\pi \gamma / 2$, in the same way as in the Gevrey case (see Theorem 1.1).

Moreover, the constant 2 in $\delta /(2 \omega)$ could be any number greater than 1 and, by suitably choosing the value $\varepsilon$ in the proof, the constant $2 B / A$ appearing before can be made as close to $B / A$ as desired, so that the only indeterminacy in the previous factor is caused by the values $A, B$ involved in (2.5). In the common situation that the function $d_{\mathbb{M}}$ is indeed a proximate order, the constants $A$ and $B$ can also be taken as near to 1 as wanted, which makes the expression even more explicit.

Finally, note that, by using Theorem 2.28 one may change $\mathbb{M}$ by an equivalent sequence $\mathbb{L}$ such that $d_{\mathbb{L}}$ is a proximate order. However, this fact does not improve the proof, since again Theorem 2.26 will be applied to obtain a function $V \in \operatorname{MF}\left(2 \omega, d_{\mathbb{L}}\right)$, and we will work with the same type of estimate that we have in (2.5).

The following lemma shows that imposing $\gamma<\omega(\mathbb{M})$ is only a technical condition in order to apply Phragmén-Lindelöf theorem 3.1.

Lemma 3.4 Let $\mathbb{M}$ and $\gamma>0$ be given. Suppose $f$ is a bounded holomorphic function in $S_{\gamma}$ that admits a continuous extension to the boundary $\partial S_{\gamma}$, and that is $\mathbb{M}$-flat in direction $d=\pi \gamma / 2$. Then for every $0<\delta<\pi \gamma$, there exist constants $k_{1}(\delta), k_{2}(\delta)>$ 0 with

$$
|f(z)| \leq k_{1} \mathrm{e}^{-M\left(1 /\left(k_{2}|z|\right)\right)}, \quad \arg z \in[-\pi \gamma / 2+\delta, \pi \gamma / 2] .
$$


Proof For simplicity, we write $\omega=\omega(\mathbb{M})$, and put $\theta_{0}:=\pi \gamma / 2$. We can obviously choose a suitable natural number $m$ and directions $\theta_{j} \in(-\pi \gamma / 2, \pi \gamma / 2)$, $j=1,2, \ldots, m$, such that

$$
\begin{aligned}
& \theta_{j}:=\theta_{j-1}-\pi \omega / 2, \quad \theta_{j} \geq-\pi \gamma / 2+\delta, \quad j=1, \ldots, m-1, \\
& \theta_{m} \in(-\pi \gamma / 2,-\pi \gamma / 2+\delta), \quad \theta_{m-1}-\theta_{m}<\pi \omega / 2 .
\end{aligned}
$$

We fix $0<\varepsilon<\pi \omega / 4$. Since $\theta_{0}-\theta_{1}+\varepsilon<3 \pi \omega / 4<\pi \omega$, we can apply Lemma 3.2 to the function $f$ restricted to the sector $\left.S_{1}=\left\{z \in \mathcal{R}: \arg z \in\left[\theta_{1}-\varepsilon, \theta_{0}\right]\right)\right\}$. We deduce that there exist constants $k_{1,1}, k_{2,1}>0$ with

$$
|f(z)| \leq k_{1,1} \mathrm{e}^{-M\left(1 /\left(k_{2,1}|z|\right)\right)}, \quad \arg z \in\left[\theta_{1}, \theta_{0}\right] .
$$

By recursively reasoning in the sectors

$$
\left.S_{j}=\left\{z \in \mathcal{R}: \arg z \in\left[\theta_{j}-\varepsilon, \theta_{j-1}\right]\right)\right\}, \quad j=2,3, \ldots, m-1,
$$

and finally in the sector

$$
\left.S_{m}=\left\{z \in \mathcal{R}: \arg z \in\left[\theta_{m}, \theta_{m-1}\right]\right)\right\},
$$

we obtain constants $k_{1, j}, k_{2, j}>0$ such that

$$
|f(z)| \leq k_{1, j} \mathrm{e}^{-M\left(1 /\left(k_{2, j}|z|\right)\right)}, \quad \arg z \in\left[\theta_{j}, \theta_{j-1}\right] .
$$

It is clear then that for $k_{1}:=\max _{j} k_{1, j}$ and $k_{2}:=\max _{j} k_{2, j}$, we have that

$$
|f(z)| \leq k_{1} \mathrm{e}^{-M\left(1 /\left(k_{2}|z|\right)\right)}, \quad \arg z \in[-\pi \gamma / 2+\delta, \pi \gamma / 2]
$$

In the next result, we impose $\mathbb{M}$-flatness in both boundary directions of the sector, and conclude uniform $\mathbb{M}$-flatness throughout the sector.

Lemma 3.5 Let $\mathbb{M}$ and $\gamma>0$ be given. Suppose $f$ is a bounded holomorphic function in $S_{\gamma}$ that admits a continuous extension to the boundary $\partial S_{\gamma}$, and that is $\mathbb{M}$-flat in directions $d=\pi \gamma / 2$ and $-d$. Then there exist constants $k_{1}, k_{2}>0$ with

$$
|f(z)| \leq k_{1} \mathrm{e}^{-M\left(1 /\left(k_{2}|z|\right)\right)}, \quad \arg z \in[-\pi \gamma / 2, \pi \gamma / 2] .
$$

Proof By Lemma 3.4, there exist constants $k_{1,1}, k_{2,1}, k_{1,2}, k_{2,2}>0$ such that

$$
|f(z)| \leq k_{1,1} \mathrm{e}^{-M\left(1 /\left(k_{2,1}|z|\right)\right)}, \quad \arg z \in[0, \pi \gamma / 2]
$$

and

$$
|f(z)| \leq k_{1,2} \mathrm{e}^{-M\left(1 /\left(k_{2,2}|z|\right)\right)}, \quad \arg z \in[-\pi \gamma / 2,0] .
$$


We conclude taking $k_{1}:=\max \left\{k_{1,1}, k_{1,2}\right\}$ and $k_{2}:=\max \left\{k_{2,1}, k_{2,2}\right\}$.

Remark 3.6 By carefully inspecting its proof, we see that Lemma 3.2 holds true in any bounded sector $S(d, \gamma, r)$ and, consequently, Lemmas 3.4 and 3.5 are also valid in bounded sectors.

We show next that, as Remark 3.6 suggests, it is also possible to work in sectorial regions.

Proposition 3.7 Let $\mathbb{M}$ and $\gamma>0$ be given. Suppose $f$ is holomorphic in a sectorial region $G_{\gamma}$, bounded in every $T \ll G_{\gamma}$, and $\mathbb{M}$-flat in a direction $\theta$ in $G_{\gamma}$. Then, for every $T \ll G_{\gamma}$ there exist constants $k_{1}(T), k_{2}(T)>0$ with

$$
|f(z)| \leq k_{1} \mathrm{e}^{-M\left(1 /\left(k_{2}|z|\right)\right)}, \quad z \in T .
$$

Proof By suitably enlarging the opening of the subsector, we can assume that $\theta$ is one of the directions in $T$. There exist $R, c_{1}, c_{2}>0$ with

$$
|f(z)| \leq c_{1} \mathrm{e}^{-M\left(1 /\left(c_{2}|z|\right)\right)}, \quad \arg z=\theta, \quad|z| \leq R
$$

If $\theta_{1}<\theta_{2}$ are the (radial) boundary directions of $T$, we consider $\delta>0$ such that $-\pi \gamma / 2<\theta_{1}-\delta$ and $\theta_{2}+\delta<\pi \gamma / 2$. There exists $0<r<R$ such that the sectors $S_{1}=\left\{z \in \mathcal{R}:|z| \leq r, \arg z \in\left[\theta_{1}-\delta, \theta\right]\right\}$ and $S_{2}=\{z \in \mathcal{R}:|z| \leq r, \arg z \in$ $\left.\left[\theta, \theta_{2}+\delta\right]\right\}$ are contained in $G_{\gamma}$. Taking into account (3.7) and Remark 3.6, we can apply Lemma 3.4 to the restriction of $f$ to each sector, and we conclude that $f$ is $\mathbb{M}$-flat for $\arg z \in\left[\theta_{1}, \theta_{2}\right]$ and $|z| \leq r$. Since $M$ is nondecreasing, by suitably enlarging the constant $k_{1}$ we obtain (3.6).

Example 3.8 Boundedness of the considered function is necessary in any of the previous results in this section. The next example shows that having an $\mathbb{M}$-asymptotic expansion in a direction $d$ does not guarantee its validity in any sector containing that direction. Our inspiration comes from a similar example in Wasow's book [24, p. 38], which concerned the function $f(z)=\sin \left(\mathrm{e}^{1 / z}\right) \mathrm{e}^{-1 / z}$.

Given $\mathbb{M}$, by Remark 2.30 for every $\gamma>0$, there exists $V \in \operatorname{MF}(\gamma, \rho)$ such that we have (2.5). We consider the function

$$
f(z)=\sin \left(\mathrm{e}^{V(1 / z)}\right) \mathrm{e}^{-V(1 / z)} \quad z \in S_{\gamma}
$$

Since $\sin \left(\mathrm{e}^{V(1 / z)}\right)$ is bounded for real $z>0$, we see that $f$ is $\mathbb{M}$-flat in direction 0 . If we compute the derivative of $f$ in $S_{\gamma}$, we see that

$$
\begin{aligned}
f^{\prime}(z) & =\frac{V^{\prime}(1 / z)}{z^{2}}\left(\sin \left(\mathrm{e}^{V(1 / z)}\right) \mathrm{e}^{-V(1 / z)}-\cos \left(\mathrm{e}^{V(1 / z)}\right)\right) \\
& =\frac{V^{\prime}(1 / z)}{z V(1 / z)} \frac{V(1 / z)}{z}\left(\sin \left(\mathrm{e}^{V(1 / z)}\right) \mathrm{e}^{-V(1 / z)}-\cos \left(\mathrm{e}^{V(1 / z)}\right)\right) .
\end{aligned}
$$


Since for $z>0$ we have $\lim _{z \rightarrow 0}(1 / z) V^{\prime}(1 / z) / V(1 / z)=1 / \omega(\mathbb{M})$ (by property (VI), see [13, Proposition 1.2]) and $\lim _{z \rightarrow 0} V(1 / z) / z=\infty$ [property (III)], we deduce that $\lim _{z \rightarrow 0} f^{\prime}(z)$ does not exist. By Remark 2.12, $f$ cannot have $\mathbb{M}$-asymptotic expansion in any sectorial region containing direction 0 . Consequently, $f$ is not $\mathbb{M}$-flat in any such sectorial region. We note that, in particular, the example of Wasow corresponds to the Gevrey case of order 1 , i.e., to the sequence $\mathbb{M}=(p !)_{p \in \mathbb{N}_{0}}$.

Remark 3.9 At this point it is worth saying a few words about a situation which, although not usually considered in the theory of asymptotic expansions, plays an important role in the general framework of ultradifferentiable or ultraholomorphic classes, namely that of the so-called Carleman classes of Beurling type. We will not give full details here, but let us say that a function $f$, holomorphic in a sectorial region $G$, has Beurling $\mathbb{M}$-asymptotic expansion $\hat{f}=\sum_{n=0}^{\infty} a_{n} z^{n}$ in a direction $\theta$ in $G$ if there exists $r_{\theta}>0$ such that the segment $\left(0, r_{\theta} \mathrm{e}^{i \theta}\right]$ is contained in $G$, and for every $A_{\theta}>0$ (small) there exists $C_{\theta}>0$ (large) such that for every $z \in\left(0, r_{\theta} \mathrm{e}^{i \theta}\right]$ and every $p \in \mathbb{N}_{0}$ one has

$$
\left|f(z)-\sum_{n=0}^{p-1} a_{n} z^{n}\right| \leq C_{\theta} A_{\theta}^{p} M_{p}|z|^{p} .
$$

Following the idea in Remark 2.23, one can prove that $f$, bounded throughout $G$, is Beurling $\mathbb{M}$-flat in direction $\theta$ if, and only if, for every $c_{2}>0$ (small) there exist $c_{1}>0$ (large) such that for every $z \in G$ with $\arg z=\theta$ one has

$$
|f(z)| \leq c_{1} \mathrm{e}^{-M\left(1 /\left(c_{2}|z|\right)\right)}
$$

Then, the following analog of Lemma 3.2 is valid: given $\mathbb{M}$ and $0<\gamma<\omega(\mathbb{M})$, suppose $f$ is a bounded holomorphic function in $S_{\gamma}$ that admits a continuous extension to the boundary $\partial S_{\gamma}$, and that is Beurling $\mathbb{M}$-flat in direction $d=\pi \gamma / 2$. Then for every $0<\delta<\pi \gamma$ and every $k_{2}>0$, there exists a constant $k_{1}=k_{1}\left(\delta, k_{2}\right)>0$ such that

$$
|f(z)| \leq k_{1} \mathrm{e}^{-M\left(1 /\left(k_{2}|z|\right)\right)}, \quad \arg z \in[-\pi \gamma / 2+\delta, \pi \gamma / 2]
$$

The proof of this statement follows the same lines as that of the original lemma, by carefully tracing the dependence of the different constants involved in the estimates. Indeed, the constants $A, B, \alpha, \beta, \varepsilon, \eta$ are determined in the same way. Choose $r_{2}>0$ such that $r_{2} / B>k_{2}^{-1 / \omega}$, and a point $a$ with the specified argument and modulus $\left(r_{2} / \eta\right)^{\omega}$. Take a positive $d_{2}$ such that $d_{2}>2|a|^{1 / \omega} / A$, and then $c_{2}>0$ such that $c_{2}<d_{2}^{-\omega}$. By definition of Beurling $\mathbb{M}$-flatness in direction $\gamma \pi / 2$, there exists $c_{1}>0$ such that (3.8) holds for $\arg z=\gamma \pi / 2$. Then, the desired estimates hold for the same $k_{1}>0$ obtained in the proof of that lemma.

Note that also Lemmas 3.4, 3.5 and Proposition 3.7 will be valid in this Beurling setting. 


\section{Watson's Lemmas}

We will now obtain several quasianalyticity results by combining those in Sects. 2.2 and 2.3 with the results on the propagation of null asymptotics in Sect. 3.

Remark 4.1 In a similar way as in the proof of Theorem 2.22 (see [21]), it is easy to deduce that, given a bounded holomorphic function $f$ in a sector $S_{\gamma}$ that admits a continuous extension to the boundary $\partial S_{\gamma}$, the fact that $f \in \tilde{\mathcal{A}}_{\mathbb{M}}^{\mathrm{u}}\left(S_{\gamma}\right)$ and $f$ is $\mathbb{M}$-flat amounts to the existence of constants $k_{1}, k_{2}>0$ such that (3.5) holds.

In the first version, an immediate consequence of previous information, we assume the function is flat at both boundary directions.

Lemma 4.2 Let $\mathbb{M}$ and $\gamma>0$ be given, such that either $\gamma>\omega(\mathbb{M})$, or $\gamma=\omega(\mathbb{M})$ and $\sum_{p=0}^{\infty}\left(m_{p}\right)^{-1 / \omega(\mathbb{M})}$ diverges. Suppose $f$ is a bounded holomorphic function in $S_{\gamma}$ that admits a continuous extension to the boundary $\partial S_{\gamma}$, and that is $\mathbb{M}$-flat in directions $d=\pi \gamma / 2$ and $-d$. Then $f \equiv 0$.

Proof By Lemma 3.5, we know that (3.5) holds for suitable $k_{1}, k_{2}>0$. The previous remark implies that $f \in \tilde{\mathcal{A}}_{\mathbb{M}}^{\mathrm{u}}\left(S_{\gamma}\right)$ and $f \sim_{\mathbb{M}} \hat{0}$, and by Corollary 2.20 we deduce that $f \equiv 0$.

In the second, improved version, we assume only that the function is flat in one of the boundary directions.

Lemma 4.3 Assume the same hypotheses as in Lemma 4.2, except that now $f$ is $\mathbb{M}$-flat only in direction $d=\pi \gamma / 2$. Then $f \equiv 0$.

Proof For simplicity, we write $\omega=\omega(\mathbb{M})$. The argument is simple if $\gamma>\omega$ : we fix $\omega<\mu<\gamma$ and $\delta=(\gamma-\mu) \pi>0$. By Lemma 3.4, we know that there exist constants $k_{1}(\delta), k_{2}(\delta)>0$ with

$$
|f(z)| \leq k_{1} \mathrm{e}^{-M\left(1 /\left(k_{2}|z|\right)\right)}, \quad \arg z \in[\pi \gamma / 2-\mu \pi, \pi \gamma / 2]
$$

Then, Remark 4.1 implies that $f \in \tilde{\mathcal{A}}_{\mathbb{M}}^{\mathrm{u}}(S)$, with $S=\{z \in \mathcal{R}: \arg z \in(\pi \gamma / 2-$ $\mu \pi, \pi \gamma / 2)\}$ and $f \sim_{\mathbb{M}} \hat{0}$. Since $\mu>\omega$, we can apply Corollary 2.20 to the function $f$ in $S$ (see also Remark 2.33), and we deduce that $f \equiv 0$.

If $\gamma=\omega$, we fix $\delta=\pi \omega / 8>0$. Lemma 3.4 ensures there exist $k_{1}(\delta), k_{2}(\delta)>0$ with

$$
|f(z)| \leq k_{1} \mathrm{e}^{-M\left(1 /\left(k_{2}|z|\right)\right)}, \quad \arg z \in[-3 \pi \omega / 8, \pi \omega / 2] .
$$

As in the proof of Lemma 3.2, since $\mathbb{M}$ admits a nonzero proximate order $\rho$, there exist $V \in \operatorname{MF}(2 \omega, \rho)$ and positive constants $A, B, t_{0}$ such that we have (2.5). Choose $q_{2}>0$ such that $k_{2}^{-1 / \omega}>q_{2}$, and take $a \in \mathcal{R}$ such that

$$
\arg a=\frac{\omega \pi}{4}, \quad 0<|a|<\left(\frac{A q_{2}}{2}\right)^{\omega} .
$$


We observe that for every $z$ with $\arg z \in[-\pi \omega / 2, \pi \omega / 2]$ one has

$$
\arg a / z \in[-\pi \omega / 4,3 \pi \omega / 4] \subseteq(-\pi \omega / 2, \pi \omega) .
$$

Using property (I) of the functions in $\operatorname{MF}(2 \omega, \rho)$, we see that

$$
\lim _{|z| \rightarrow 0} \Re\left(\frac{V(a / z)}{|a|^{1 / \omega} V(1 /|z|)}\right)=\cos ((\arg a-\arg z) / \omega)
$$

uniformly for $\arg z \in[-\pi \omega / 2, \pi \omega / 2]$. We fix $0<\varepsilon<1$ such that

$$
\cos 3 \pi / 4+\varepsilon \leq \cos 5 \pi / 8+\varepsilon \leq-1 / 3<0 .
$$

We deduce that we have (3.3) and (3.4) for $\arg z \in[-\pi \omega / 2, \pi \omega / 2]$ and $|z|<s_{1}$, small enough and subject to the restriction $s_{1}<1 /\left(t_{0} k_{2}\right)$. Consider the function

$$
F(z):=f(z) \mathrm{e}^{V(a / z)}, \quad \arg z \in[-\pi \omega / 2, \pi \omega / 2] .
$$

Then we see that $F$ is holomorphic in $S_{\omega}$ and continuous in $\overline{S_{\omega}}$.

If $\arg z \in[-\pi \omega / 2,-3 \pi \omega / 8]$, we have that $\arg a / z \in[5 \pi \omega / 8,3 \pi \omega / 4]$. Then, since $f$ is bounded by $K>0$ in $\overline{S_{\omega}}$ and using (3.4) for $|z|<s_{1}$, one has

$$
|F(z)| \leq K \mathrm{e}^{\Re(V(a / z))} \leq K \mathrm{e}^{(\cos 5 \pi / 8+\varepsilon)|a|^{1 / \omega} V(1 /|z|)} \leq K \mathrm{e}^{-|a|^{1 / \omega} V(1 /|z|) / 3} .
$$

Using property (I) of the functions in $\operatorname{MF}(2 \omega, \rho)$ we see that

$$
\lim _{|z| \rightarrow 0} \frac{V\left(\left(|a| /(3 B)^{\omega}\right)(1 / 2|z|)\right)}{\left(|a|^{1 / \omega} /(3 B)\right) V(1 /|z|)}=(1 / 2)^{1 / \omega}<1 .
$$

We define $b_{2}:=\left(|a| /(3 B)^{\omega}\right) / 2$. Then for $|z|<s_{2}<\min \left(s_{1}, b_{2} / t_{0}\right)$, small enough, we have that

$$
|F(z)| \leq K \mathrm{e}^{-B V\left(b_{2} /|z|\right)}, \quad|z|<s_{2}, \quad \arg z \in[-\pi \omega / 2,-3 \pi \omega / 8]
$$

Using (2.5), we see that

$$
|F(z)| \leq K \mathrm{e}^{-M\left(b_{2} /|z|\right)}, \quad|z|<s_{2}, \quad \arg z \in[-\pi \omega / 2,-3 \pi \omega / 8] .
$$

We define $C=\max \left\{\Re(V(a / z)):|z| \geq s_{2},-\pi \omega / 2 \leq \arg z \leq-3 \pi \omega / 8\right\}$ and we take

$$
c_{1}:=K \max \{\exp (C), 1\}<\infty
$$

Then, since $M(t) \geq 0$, we have that

$$
|F(z)| \leq c_{1} \leq c_{1} e^{M\left(b_{2} /|z|\right)} \quad|z| \geq s_{2}, \quad \arg z \in[-\pi \omega / 2,-3 \pi \omega / 8] .
$$


Since $c_{1} \geq K$, from (4.2) and (4.3), we deduce that $F$ is $\mathbb{M}$-flat for $\arg z \in$ $[-\pi \omega / 2,-3 \pi \omega / 8]$.

If $\arg z \in[-3 \pi \omega / 8, \pi \omega / 2]$, we have that $\arg a / z \in[-\pi \omega / 4,5 \pi \omega / 8]$. Using (2.5), (3.4) and (4.1), for $|z|<s_{1}$, we see that

$$
|F(z)| \leq k_{1} \mathrm{e}^{-M\left(1 /\left(k_{2}|z|\right)\right)} \mathrm{e}^{(\cos (\arg (a / z) / \omega)+\varepsilon)|a|^{1 / \omega} V(1 /|z|)} \leq k_{1} \mathrm{e}^{-A V\left(1 / k_{2}|z|\right)+2|a|^{1 / \omega} V(1 /|z|)} .
$$

Now, property (I) of the functions in $\operatorname{MF}(2 \omega, \rho)$ lets us write

$$
\lim _{|z| \rightarrow 0} \frac{V\left(1 / k_{2}|z|\right)}{V(1 /|z|)}=k_{2}^{-1 / \omega}
$$

so that, for $|z|<s_{3} \leq s_{2}$ small enough, we have that $V\left(1 / k_{2}|z|\right) \geq q_{2} V(1 /|z|)$. We conclude that

$$
|F(z)| \leq k_{1} \mathrm{e}^{\left(-A q_{2}+2|a|^{1 / \omega}\right) V(1 /|z|)}, \quad|z|<s_{3}, \quad \arg z \in[-3 \pi \omega / 8, \pi \omega / 2] .
$$

Since $|a|$ has been chosen small enough in order that $-A q_{2}+2|a|^{1 / \omega}<0$, proceeding as before, we find that $F$ is $\mathbb{M}$-flat for $\arg z \in[-3 \pi \omega / 8, \pi \omega / 2]$.

Consequently, $F$ verifies estimates of the type (3.5) in $\overline{S_{\omega}}$ and, by Remark 4.1, $F \in \tilde{\mathcal{A}}_{\mathbb{M}}^{\mathrm{u}}\left(S_{\omega}\right)$ and $F \sim_{\mathbb{M}} \hat{0}$. Since $\sum_{p=0}^{\infty}\left(m_{p}\right)^{-1 / \omega(\mathbb{M})}$ is assumed to be divergent, we can apply Corollary 2.20 to the function $F$ in $S_{\omega}$, and deduce that $F \equiv 0$ and $f \equiv 0$.

In the proof of Lemma 4.3, we need to distinguish two situations: in case $\gamma>$ $\omega(\mathbb{M})$, we have been given an $\mathbb{M}$-flat function $f$ in a wide enough sector (what entails uniqueness), while in case $\gamma=\omega(\mathbb{M})$ an $\mathbb{M}$-flat function $F$ in a sector of opening $\pi \omega(\mathbb{M})$ has to be constructed in order to apply Corollary 2.20, what is possible thanks to the additional assumption on the series $\sum_{p=0}^{\infty}\left(m_{p}\right)^{-1 / \omega(\mathbb{M})}$.

It is interesting to note that in the Gevrey case the aforementioned series diverges, so that the previous result extends Lemma 5 in [3]. Indeed, in that instance the very divergence of the series allows one to treat the case $\gamma>\omega(\mathbb{M})$ by restricting the function to a sector with $\gamma=\omega(\mathbb{M})$, an argument which is not available in our situation.

Remark 4.4 In most situations, we can obtain converse statements to Lemmas 4.2 and 4.3. Observe that if $\gamma<\omega(\mathbb{M})$ and we take $\gamma<\mu<\omega(\mathbb{M})$, by Corollary 2.20, we know there exists a nontrivial $\mathbb{M}$-flat function $f \in \tilde{\mathcal{A}}_{\mathbb{M}}^{\mathrm{u}}\left(S_{\mu}\right)$. Then (the restriction of) $f$ is a bounded holomorphic function in $S_{\gamma}$ that admits a continuous extension to the boundary $\partial S_{\gamma}$, and that is $\mathbb{M}$-flat in directions $d=\pi \gamma / 2$ and $-d$.

Analogously, if $\gamma=\omega$ and $\sum_{p=0}^{\infty}\left((p+1) m_{p}\right)^{-1 /(\omega(\mathbb{M})+1)}$ converges, we deduce that $\sum_{p=0}^{\infty}\left(m_{p}\right)^{-1 / \omega(\mathbb{M})}$ converges too. So, by Corollary 2.17 there exists a nontrivial $\mathbb{M}$-flat function $f \in \mathcal{A}_{\mathbb{M}}\left(S_{\omega(\mathbb{M})}\right)$. Since the derivatives of $f$ are Lipschitzian, one may continuously extend $f$ to the boundary of $S_{\omega(\mathbb{M})}$ preserving the estimates, and again obtain that $f$ is $\mathbb{M}$-flat in directions $\pi \omega(\mathbb{M}) / 2$ and $-\pi \omega(\mathbb{M}) / 2$.

However, the converse of Lemmas 4.2 and 4.3 fails in case $\gamma=\omega(\mathbb{M})$, the series $\sum_{p=0}^{\infty}\left(m_{p}\right)^{-1 / \omega(\mathbb{M})}$ converges and $\sum_{p=0}^{\infty}\left((p+1) m_{p}\right)^{-1 /(\omega(\mathbb{M})+1)}$ diverges 
[for instance, this is the situation for the sequence $\mathbb{M}_{1,3 / 2}$, see the Examples 2.9(i)]. Although nontrivial $\mathbb{M}$-flat functions in $\tilde{\mathcal{A}}_{\mathbb{M}}^{\mathrm{u}}\left(S_{\omega(\mathbb{M})}\right)$ exist in this situation, there is no warranty that they can be continuously extended to the boundary of the sector.

Finally, we provide a version of Watson's Lemma for functions in sectorial regions which are flat in a direction.

Proposition 4.5 Let $\mathbb{M}$ and $\gamma>0$ be given with $\gamma>\omega(\mathbb{M})$. Suppose $f$ is holomorphic in a sectorial region $G_{\gamma}$, bounded in every $T \ll G_{\gamma}$, and $\mathbb{M}$-flat in a direction $\theta$ in $G_{\gamma}$. Then $f \equiv 0$.

Proof Using Proposition 3.7 we know that for every $T \ll G_{\gamma}$, we have (3.6) for suitable $k_{1}, k_{2}>0$ depending on $T$ and for every $z \in T$. Then, Theorem 2.22 implies that $f \in \tilde{\mathcal{A}}_{\mathbb{M}}\left(G_{\gamma}\right)$ and $f \sim_{\mathbb{M}} \hat{0}$, and Theorem 2.32 leads to the conclusion.

Remark 4.6 By Theorem 2.32, if $\gamma \leq \omega$, we can find a nontrivial function $f \in$ $\tilde{\mathcal{A}}_{\mathbb{M}}\left(G_{\gamma}\right)$ such that $f \sim_{\mathbb{M}} \hat{0}$, so it is bounded on every proper bounded subsector $T$ of $G_{\gamma}$ and $\mathbb{M}$-flat in any direction $\theta_{0} \in(-\pi \gamma / 2, \pi \gamma / 2)$. Consequently, in this situation we have a complete version of Watson's Lemma.

\section{Asymptotic Expansion Extension}

The next result (see [18, Theorem 6.1]) was stated for strongly regular sequences $\mathbb{M}$ such that $d_{\mathbb{M}}$ is a proximate order. However, as it is deduced from [18, Remark 4.11(iii)] and [9, Remark 4.15], it is enough to ask for the sequence to satisfy our two general assumptions (see Sect. 3).

Theorem 5.1 (Generalized Borel-Ritt-Gevrey theorem) Let $\mathbb{M}$ and $\gamma>0$ be given. The following statements are equivalent:

(i) $\gamma \leq \omega(\mathbb{M})$,

(ii) For every $\hat{f}=\sum_{p \in \mathbb{N}_{0}} a_{p} z^{p} \in \mathbb{C}[[z]]_{\mathbb{M}}$, there exists a function $f \in \tilde{\mathcal{A}}_{\mathbb{M}}\left(S_{\gamma}\right)$ such that

$$
f \sim_{\mathbb{M}} \hat{f}
$$

i.e., $\tilde{\mathcal{B}}(f)=\hat{f}$. In other words, the Borel map $\tilde{\mathcal{B}}: \tilde{\mathcal{A}}_{\mathbb{M}}\left(S_{\gamma}\right) \longrightarrow \mathbb{C}[[z]]_{\mathbb{M}}$ is surjective.

From this result, we may generalize Theorem 1 in [3].

Theorem 5.2 Given $\mathbb{M}$ and $\gamma>0$, suppose $f$ is holomorphic in a sectorial region $G_{\gamma}$, is bounded in every $T \ll G_{\gamma}$, and it admits $\hat{f} \in \mathbb{C}[[z]]$ as its $\mathbb{M}$-asymptotic expansion in a direction $\theta \in(-\pi \gamma / 2, \pi \gamma / 2)$. Then, $f \in \tilde{\mathcal{A}}_{\mathbb{M}}\left(G_{\gamma}\right)$ and $f \sim_{\mathbb{M}} \hat{f}$ in $G_{\gamma}$.

Proof We distinguish two cases: 
(1) Sectorial regions of small opening: if $\gamma<\omega$, we take $\gamma<\mu<\omega$. By the BorelRitt-Gevrey Theorem 5.1, we know that there exists a function $f_{0} \in \tilde{\mathcal{A}}_{\mathbb{M}}\left(S_{\mu}\right)$ such that $f_{0} \sim_{\mathbb{M}} \hat{f}$ in $S_{\mu}$. Then the function $g:=f-f_{0}$ is holomorphic in $G_{\gamma}$, bounded in every proper bounded subsector of $G_{\gamma}$ and it is $\mathbb{M}$-flat in direction $\theta$. Using Proposition 3.7, we see that $g$ is $\mathbb{M}$-flat in $G_{\gamma}$.

Then, for every proper bounded subsector $T$ of $G_{\gamma}$, there exists positive constants $A(T), B(T), C(T), D(T)>0$ such that

$$
\begin{aligned}
\left|f(z)-\sum_{n=0}^{p-1} a_{n} z^{n}\right| & \leq|g(z)|+\left|f_{0}(z)-\sum_{n=0}^{p-1} a_{n} z^{n}\right| \\
& \leq A C^{p} M_{p}|z|^{p}+B D^{p} M_{p}|z|^{p} \\
& \leq 2 \max (A, B) \max \left(C^{p}, D^{p}\right) M_{p}|z|^{p}
\end{aligned}
$$

for every $z \in T$ and every $p \in \mathbb{N}_{0}$. Consequently, $f \in \tilde{\mathcal{A}}_{\mathbb{M}}\left(G_{\gamma}\right)$ and $f \sim_{\mathbb{M}} \hat{f}$ in $G_{\gamma}$.

(2) Sectorial regions of large opening: if $\gamma \geq \omega$, we may choose natural numbers $\ell$ and $m$, and for $j=-\ell, \ldots,-1,0,1,2, \ldots, m$, we may consider directions $\theta_{j} \in(-\pi \gamma / 2, \pi \gamma / 2)$ such that

$$
\begin{aligned}
& \theta_{0}:=\theta, \quad \theta_{j}:=\theta_{j-1}+\pi \omega / 8, \quad j=1, \ldots, m, \quad \pi \gamma / 2-\theta_{m}<\pi \omega / 8 \\
& \theta_{j}:=\theta_{j+1}-\pi \omega / 8, \quad j=-1, \ldots,-l, \quad-\pi \gamma / 2+\theta_{-l}>-\pi \omega / 8
\end{aligned}
$$

There exists $\rho_{0}>0$ such that $S_{0}=S\left(\theta_{0}, \pi \omega / 4, \rho_{0}\right) \subseteq G_{\gamma}$. We apply the first part in the sector $S_{0}$ and we see that $f \in \tilde{\mathcal{A}}_{\mathbb{M}}\left(S_{0}\right)$ and $f \sim_{\mathbb{M}} \hat{f}$ in $S_{0}$. In particular, $f$ admits $\hat{f}$ as its $\mathbb{M}$-asymptotic expansion in directions $\theta_{1}$ and $\theta_{-1}$ for $|z|<\rho_{0}$. Repeating the process, we see that $f \in \tilde{\mathcal{A}}_{\mathbb{M}}\left(G_{\gamma}\right)$ and $f \sim_{\mathbb{M}} \hat{f}$ in $G_{\gamma}$.

The proof of our last statement is now straightforward.

Corollary 5.3 Given $\mathbb{M}, \gamma>0$ and $\theta \in(-\pi \gamma / 2, \pi \gamma / 2)$, we have that

$$
\begin{array}{r}
\tilde{\mathcal{A}}_{\mathbb{M}}\left(G_{\gamma}\right)=\left\{f \in \mathcal{H}\left(G_{\gamma}\right): f \text { is bounded in every proper bounded subsector } T \text { of } G_{\gamma}\right. \\
\text { and } f \text { admits } \mathbb{M} \text {-asymptotic expansion in direction } \theta\} .
\end{array}
$$

Acknowledgements Open access funding provided by Austrian Science Fund (FWF). The first two authors are partially supported by the Spanish Ministry of Economy and Competitiveness under Project MTM201677642-C2-1-P. The first author is partially supported by the University of Valladolid through a Predoctoral Fellowship (2013 Call) cosponsored by the Banco de Santander. The third author is supported by the FWFProject J 3948-N35, as a part of which he has been an External Researcher at the Universidad de Valladolid (Spain) for the period October 2016-December 2018. Part of these results were obtained in the course of a research stay of the first author in the Centro di Ricerca Matematica Ennio De Giorgi (Scuola Normale Superiore di Pisa, Italy), for which the first author expresses his gratitude.

Open Access This article is distributed under the terms of the Creative Commons Attribution 4.0 International License (http://creativecommons.org/licenses/by/4.0/), which permits unrestricted use, distribution, and reproduction in any medium, provided you give appropriate credit to the original author(s) and the source, provide a link to the Creative Commons license, and indicate if changes were made. 


\section{References}

1. Balser, W.: From Divergent Power Series to Analytic Functions. Lecture Notes in Mathematics, vol. 1582. Springer, New York (1994)

2. Canalis-Durand, M.: Asymptotique Gevrey. http://www.dance-net.org/files/events/rtns2010/ materiales/Canalis.pdf. Accessed 23 Aug 2018

3. Fruchard, A., Zhang, C.: Remarques sur les développements asymptotiques. Ann. Fac. Sci. Toulouse Math. (6) 8(1), 91-115 (1999)

4. Galindo, F., Sanz, J.: On strongly asymptotically developable functions and the Borel-Ritt theorem. Studia Math. 133(3), 231-248 (1999)

5. Haraoka, Y.: Theorems of Sibuya-Malgrange type for Gevrey functions of several variables. Funkcial. Ekvac. 32, 365-388 (1989)

6. Holland, A.S.B.: Introduction to the Theory of Entire Functions. Academic, New York (1973)

7. Jiménez-Garrido, J.: Applications of regular variation and proximate orders to ultraholomorphic classes, asymptotic expansions and multisummability, PhD Dissertation, University of Valladolid (2018). http://uvadoc.uva.es/handle/10324/29501. Accessed 23 Aug 2018

8. Jiménez-Garrido, J., Sanz, J.: Strongly regular sequences and proximate orders. J. Math. Anal. Appl. 438(2), 920-945 (2016)

9. Jiménez-Garrido, J., Sanz, J., Schindl, G.: Log-convex sequences and nonzero proximate orders. J. Math. Anal. Appl. 448(2), 1572-1599 (2017)

10. Korenbljum, B.I.: Conditions of nontriviality of certain classes of functions analytic in a sector, and problems of quasianalyticity. Sov. Math. Dokl. 7, 232-236 (1966)

11. Lastra, A., Malek, S., Sanz, J.: Summability in general Carleman ultraholomorphic classes. J. Math. Anal. Appl. 430, 1175-1206 (2015)

12. Lastra, A., Mozo-Férnandez, J., Sanz, J.: Strong asymptotic expansions in a multidirection. Funkcial. Ekvac. 55, 317-345 (2012)

13. Maergoiz, L.S.: Indicator diagram and generalized Borel-Laplace transforms for entire functions of a given proximate order. St. Petersbg Math. J. 12(2), 191-232 (2001)

14. Majima, H.: Analogues of Cartan's decomposition theorem in asymptotic analysis. Funkcial. Ekvac. 26, 131-154 (1983)

15. Majima, H.: Asymptotic Analysis for Integrable Connections with Irregular Singular Points. Lecture Notes in Mathematics, vol. 1075. Springer, Berlin (1984)

16. Mandelbrojt, S.: Séries adhérentes, régularisation des suites, applications. Collection de monographies sur la théorie des fonctions. Gauthier-Villars, Paris (1952)

17. Rodríguez-Salinas, B.: Funciones con momentos nulos. Rev. Acad. Cienc. Madr. 49, 331-368 (1955)

18. Sanz, J.: Flat functions in Carleman ultraholomorphic classes via proximate orders. J. Math. Anal. Appl. 415(2), 623-643 (2014)

19. Schmets, J., Valdivia, M.: Extension maps in ultradifferentiable and ultraholomorphic function spaces. Studia Math. 143(3), 221-250 (2000)

20. Thilliez, V.: Division by flat ultradifferentiable functions and sectorial extensions. Results Math. 44, 169-188 (2003)

21. Thilliez, V.: Smooth solutions of quasianalytic or ultraholomorphic equations. Mon. Math. 160, 443453 (2010)

22. Titchmarsh, E.C.: The Theory of Functions. Reprint of the second (1939) edition. Oxford University Press, Oxford (1958)

23. Trunov, K.V., Yulmukhametov, R.S.: Quasianalytic Carleman classes on bounded domains. St. Petersbg Math. J. 20(2), 289-317 (2009)

24. Wasow, W.: Asymptotic Expansions for Ordinary Differential Equations. Dover, New York (1987)

25. Yulmukhametov, R.S.: Quasianalytical classes of functions in convex domains. Math. USSR Sb. 58(2), 505-523 (1987)

Publisher's Note Springer Nature remains neutral with regard to jurisdictional claims in published maps and institutional affiliations. 